\title{
Prognostic role of preoperative serum lipid levels in patients undergoing radical prostatectomy for clinically localized prostate cancer
}

Wettstein, Marian S ; Saba, Karim ; Umbehr, Martin H ; Murtola, Teemu J ; Fankhauser, Christian D ; Adank, Jean-Pascal ; Hofmann, Marc ; Sulser, Tullio ; Hermanns, Thomas ; Moch, Holger ; Wild, Peter ; Poyet, Cédric

\begin{abstract}
BACKGROUND The prognostic role of preoperative serum lipid levels in patients undergoing radical prostatectomy $(\mathrm{RP})$ for clinically localized prostate cancer $(\mathrm{PCa})$ is unclear. The aim of the present study was to investigate preoperative serum lipid levels in patients with clinically localized PCa undergoing RP and their association with clinicopathological features and oncological outcome. METHODS Preoperative lipid levels (total cholesterol, LDL cholesterol, HDL cholesterol and triglycerides) and statin use from consecutive patients with clinically localized PCa undergoing RP in a tertiary referral center between 2008 and 2015 were recorded and patients were followed prospectively. Logistic regression analysis was used to test the association between lipid levels and clinicopathological parameters. Lipid values were analyzed both as continuous and dichotomized variables. Univariable and multivariable Cox regression analyses were performed to identify predictors for recurrence-free survival (RFS). Recurrence was defined as rising and verified PSA levels $>0.1 \mathrm{ng} / \mathrm{ml}$. RESULTS Our cohort consisted of 371 men with a median age of 63 years (range 41-78 years) and a median preoperative PSA value of $6.79 \mathrm{ng} / \mathrm{ml}$ (0.43-81.4 ng/ml). Median follow-up was 28 months (1-64). No association was found between lipid levels and adverse pathological characteristics such as pT3, Gleason score 8, positive nodal status and positive surgical margins. Recurrence occurred in 49 patients (15.4\%) at a median time of 18 months (2-51 month). Compared to low LDL cholesterol, high LDL cholesterol was associated with longer RFS in univariable analysis (continuous: Hazard Ratio (HR): 0.67, 95\%-Confidence Interval (CI): 0.47-0.96, P = 0.03; 3 mM cut-point: HR: 0.44, 95\%-CI: 0.24-0.79, P = 0.006). Neither levels of other lipids, nor statin use were associated with RFS. Preoperative LDL cholesterol remained an independent predictor for PCa recurrence in a multivariable model adjusted for age, preoperative PSA, statin use, tumor stage, Gleason score, nodal status and surgical margin status (continuous: HR: 0.66, 95\%-CI: 0.44-0.99, P = 0.04; $3 \mathrm{mM}$ cut-point: HR: $0.41,95 \%-C I: 0.21-0.78, \mathrm{P}=0.007)$. CONCLUSIONS This is the first prospective study showing the potential adverse and independent prognostic role of low preoperative LDL cholesterol levels in patients with localized PCa undergoing RP. Prostate (C) 2017 Wiley Periodicals, Inc.
\end{abstract}

DOI: https://doi.org/10.1002/pros.23296

Posted at the Zurich Open Repository and Archive, University of Zurich

ZORA URL: https://doi.org/10.5167/uzh-133384

Journal Article

Accepted Version

Originally published at: 
Wettstein, Marian S; Saba, Karim; Umbehr, Martin H; Murtola, Teemu J; Fankhauser, Christian D; Adank, Jean-Pascal; Hofmann, Marc; Sulser, Tullio; Hermanns, Thomas; Moch, Holger; Wild, Peter; Poyet, Cédric (2017). Prognostic role of preoperative serum lipid levels in patients undergoing radical prostatectomy for clinically localized prostate cancer. The Prostate, 77(5):549-556.

DOI: https://doi.org/10.1002/pros.23296 


\title{
Prognostic role of preoperative serum lipid levels in patients undergoing radical prostatectomy for clinically localized prostate cancer
}

\author{
MS Wettstein ${ }^{\mathrm{a}^{\circ}}, \mathrm{K} \mathrm{Saba}^{\mathrm{a}^{\circ}}$, MH Umbehr ${ }^{\mathrm{a}}$, TJ Murtola ${ }^{\mathrm{b}}$, CD Fankhauser $^{\mathrm{a}}$, JP Adank $^{\mathrm{a}}$, M

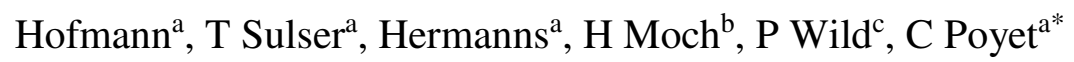

${ }^{a}$ Department of Urology, University Hospital, University of Zurich, Zurich, Switzerland bepartment of Urology, Tampere University Hospital, University of Tampere, Tampere, Finland

${ }^{\mathrm{c}}$ Department of Pathology and Molecular Pathology, University Hospital, University of Zurich, Zurich, Switzerland

*Corresponding author:

Department of Urology, University Hospital of Zurich, Frauenklinikstrasse 10, 8091 Zurich, Switzerland, Tel: +41-44-2551111, Fax: +41-44-2554566, email: cedric.poyet@usz.ch

${ }^{\circ}$ both authors contributed equally to this work

Running head: Serum lipids and biochemical recurrence

\section{Conflicts of Interest}

The authors of this article declare that they have nothing to disclose. 


\begin{abstract}
Background: The prognostic role of preoperative serum lipid levels in patients undergoing radical prostatectomy $(\mathrm{RP})$ for clinically localized prostate cancer $(\mathrm{PCa})$ is unclear. The aim of the present study was to investigate preoperative serum lipid levels in patients with clinically localized PCa undergoing RP and their association with clinicopathological features and oncological outcome.
\end{abstract}

Methods: Preoperative lipid levels (total cholesterol, LDL cholesterol, HDL cholesterol and triglycerides) and statin use from consecutive patients with clinically localized PCa undergoing RP in a tertiary referral center between 2008 and 2015 were recorded and patients were followed prospectively. Logistic regression analysis was used to test the association between lipid levels and clinicopathological parameters. Lipid values were analyzed both as continuous and dichotomized variables. Univariable and multivariable Cox regression analyses were performed to identify predictors for recurrence-free survival (RFS). Recurrence was defined as rising and verified PSA levels $>0.1 \mathrm{ng} / \mathrm{mL}$.

Results: Our cohort consisted of 371 men with a median age of 63 years (range 41-78 years) and a median preoperative PSA value of $6.79 \mathrm{ng} / \mathrm{mL}(0.43-81.4 \mathrm{ng} / \mathrm{mL})$. Median follow-up was 28 months (1-64). No association was found between lipid levels and adverse pathological characteristics such as $\geq \mathrm{pT} 3$, Gleason score $\geq 8$, positive nodal status and positive surgical margins. Recurrence occurred in 49 patients $(15.4 \%)$ at a median time of 18 months (2-51 month). Compared to low LDL cholesterol, high LDL cholesterol was associated with longer RFS in univariable analysis (continuous: Hazard Ratio (HR): 0.67, 95\%-Confidence Interval (CI): 0.47-0.96, p=0.03; 3 mM cut-point: HR: 0.44, 95\%-CI: 0.24-0.79, p=0.006). Neither levels of other lipids, nor statin use were associated with RFS. Preoperative LDL cholesterol remained an independent predictor for PCa recurrence in a multivariable model adjusted for age, preoperative PSA, statin use, tumor stage, Gleason score, nodal status and surgical margin status (continuous: HR: 0.66, 95\%-CI: 0.44-0.99, $\mathrm{p}=0.04 ; 3 \mathrm{mM}$ cut-point: HR: $0.41,95 \%-C I: 0.21-0.78, \mathrm{p}=0.007)$.

Conclusions: This is the first prospective study showing the potential adverse and independent prognostic role of low preoperative LDL cholesterol levels in patients with localized PCa undergoing RP. 


\section{Key words}

Prostate cancer, radical prostatectomy, biochemical recurrence, serum lipids 


\section{Introduction}

Prostate cancer (PCa) is one of the most commonly diagnosed cancers among men worldwide, with an estimated 1.1 million new cases and 0.3 million PCa-related deaths per year [1]. One well established treatment option for localized PCa with good long-term cancer control is radical prostatectomy (RP) [2]. Biochemical recurrence (BCR) after RP is often assumed to represent cancer and precede clinical progression. Even in specialized highvolume centers BCR rates after RP might reach up to $40 \%$ after 10 years [3].

The impact of serum lipid levels on the incidence of $\mathrm{PCa}$ and its natural course remains controversial [4,5]. A recent meta-analysis showed that total cholesterol, high-density lipoprotein (HDL) cholesterol and low-density lipoprotein (LDL) cholesterol serum levels are unlikely to be associated with PCa risk [6]. However, the role of dyslipidemia in secondary prevention as a potential modifiable risk factor for PCa recurrence is even less clear. The association between complete preoperative serum lipid status and biochemical recurrence (BCR) after curative treatment for clinically localized PCa has only been investigated in a few studies, which have reported conflicting results [7-10]. For example, one study reported elevated serum triglycerides to be associated with increased risk of PCa recurrence [7], whereas another study found an association between hypertriglyceridemia and longer BCRfree survival [8]. Based on the conflicting results of studies in PCa patients, the association of preoperative lipid levels with BCR after curative treatment for localized PCa warrants further analysis. The aim of this study was to examine the association between the complete preoperative lipid status, clinicopathological features and BCR in men with clinically localized PCa. 


\section{Materials and Methods}

Study design

Men with clinically localized PCa undergoing laparoscopic robotic assisted RP were prospectively included in this single-center cohort study (Prostate Cancer outcomes cohort study: ProCOC [11]). The study was approved by the Ethics Committee of Canton Zürich (protocol name: ProCOC: The Prostate Cancer Outcomes Cohort Study, protocol number: Ref. Nr. StV KEK-ZH-Nr. 06/08). The cohort consisted only of men with clinically localized PCa who were scheduled for RP at our tertiary referral center and gave informed consent. Patients were normally followed on a regular basis for every three months the first year and afterwards at least annually or on an individual basis depending on the disease course. A PSA value of $0.1 \mathrm{ng} / \mathrm{ml}$ or higher was defined as BCR. Patients were censored if lost to follow-up or event-free at their most recent clinic visit. Patients with a postoperative PSA persistence or without distinct follow-up data for the endpoint BCR were excluded from the analysis of BCR.

\section{Pathologic analysis}

All surgical specimens were processed according to standard histopathological procedures. At least one uropathologist assigned pathologic stage, tumor grade and node status. Surgical margins were considered positive if any neoplastic cells were in contact with the inked surface of the prostatectomy specimen. Tumor characteristics were obtained from pathology reports according to the WHO/ISUP 2016 classification.

\section{Analysis of serum lipids}

Preoperative serum lipid levels (total cholesterol, LDL cholesterol, HDL cholesterol and triglycerides) were assessed the day before surgery for all men according to the study protocol. Additionally, statin use at the time of surgery was recorded. The duration of 
preoperative statin intake was not taken into account in this study. Blood analysis was performed by the hospital's Institute of Clinical Chemistry. Total cholesterol, HDL cholesterol and triglycerides were determined by enzymatic colorimetric tests in a modular analyzer (Cobas 8000, Roche Diagnostics GmbH, Mannheim, D). Friedewald's formula ( LDL cholesterol $=$ Total cholesterol $-[$ HDL cholesterol $+\{$ triglycerides $/ 5\}])$ was used to calculate serum LDL cholesterol values [12]. However, this formula is not valid for triglyceride levels $>4.5 \mathrm{mmol} / \mathrm{L}(\mathrm{mM})$ and patients with levels above this threshold were thus excluded from any analysis using LDL cholesterol as a variable.

\section{Statistical analysis}

Univariable logistic regression analysis was used to analyse the association between serum lipid levels, statin use and clinicopathological tumor characteristics. Clinicopathological study outcomes were extraprostatic disease ( $\geq \mathrm{pT} 3$ ), high-risk disease (Gleason Score $\geq 8$ ), positive nodal status (pN1) and positive surgical margins (PSM).

Serum lipid levels in the regression analysis were analyzed as continuous and as binary variables. Cut-points were selected according to the reference values of the hospital's Institute of Clinical Chemistry that are based on internationally accepted threshold values (total cholesterol: $5 \mathrm{mM}$, LDL cholesterol: $3 \mathrm{mM}$, HDL cholesterol: $1 \mathrm{mM}$, triglycerides: $1.7 \mathrm{mM}$ ) [13].

Univariable and multivariable Cox regression models addressed the association of serum lipid levels with BCR after RP. Adjustments were made for age, preoperative PSA, statin use, tumor stage, Gleason score, nodal status and surgical margin status. We constructed Cox regression models for each single adjustment factor and one model containing all adjustment factors.

Cut-point analysis was conducted for independently prognostic serum lipid values to detect other significant dichotomization cut-points ( $0.5 \mathrm{mM}$ increments). Multiple comparisons were 
handled by adjusting the p-values with the false-discovery rate (FDR) approach described by Benjamini \& Hochberg [14]. Finally, Kaplan-Meier survival plots with their corresponding log-rank tests were drawn for all significant cut-points that were found in the cut-point analysis.

$\mathrm{R}$ programming language and software environment version 3.1.3 ( $\mathrm{R}$ Foundation for Statistical Computing, Vienna, A) was used to perform all statistical analyses. All p-values were two-sided with p-values $<0.05$ considered statistically significant. 


\section{Results}

Between 2008 and 2015, a total of 371 consecutive PCa patients before RP were included. The clinical and pathological characteristics, preoperative serum lipid levels and statin use are summarized in Table 1. A total of 108 (29.2\%) men had extraprostatic disease and $79(21.4 \%)$ had high-risk disease (Gleason score $\geq 8$ ). Furthermore, 25 (6.7\%) men were diagnosed with lymph node metastasis. Pelvic lymphadenectomy was performed in 277 (74.7\%) men. Median preoperative serum lipid levels (total cholesterol, LDL cholesterol, HDL cholesterol, triglycerides) are also presented in Table 1. LDL cholesterol was non-calculable in five patients $(1.3 \%)$ due to high cholesterol levels $(>4.5 \mathrm{mM})$. These patients were excluded from all analyses, which used LDL cholesterol as a variable. A total of $61(16.4 \%)$ men were statin users at the time of surgery. A total of 52 patients (14\%) were excluded from the analysis of time to BCR due to postoperative PSA persistence $(n=17)$ or unavailable follow-up data regarding BCR ( $n=35)$ after RP. Median follow-up time for the remaining 319 men was 28 months (range 1-64). BCR occurred in 49 patients (15.4\%) after a median time of 18 months (2-51).

Univariable logistic regression analysis (Table 2) revealed a significant association between triglycerides (continuous) and high-risk disease (OR: 1.32; 95\%-CI: 1.03-1.69; p=0.03). However, the association between triglycerides and high-risk disease could not be confirmed when triglycerides were used as a dichotomized variable (OR: 1.05; 95\%-CI: 0.63-1.75; $\mathrm{p}=0.86$ ). All other serum lipid levels (continuous and dichotomized) did not reveal any significant association with pathological tumor characteristics (Table 2). Among all pathological parameters, statin use only showed a positive association with PSM (OR: 1.94; 95\%-CI: 1.12-3.39; $\mathrm{p}=0.02$ ). In an additional univariable logistic regression analysis, preoperative serum lipid levels and statin use were not associated with high preoperative PSA values $(\geq 10 \mathrm{ng} / \mathrm{ml})$ (Table $\mathrm{S} 1)$. 
For prognostic association of serum lipid levels and statin use uni- and multivariable Cox regression analyses were performed. Among all preoperative lipid values, only high LDL (continuous and $3 \mathrm{mM}$ cut-point) cholesterol values were significantly associated with longer recurrence free survival (RFS) compared to low LDL values in univariable analysis (continuous: HR: 0.67, 95\%-CI: 0.47-0.96, p=0.03; 3 mM cut-point: HR: 0.44, 95\%-CI: 0.240.79, $\mathrm{p}=0.006$ ). Additionally, all clinicopathological study outcomes (stage $\geq \mathrm{pT} 3$, Gleason score $\geq 8, \mathrm{pN} 1$ and PSM) were highly associated with a shorter RFS (for all $\mathrm{p}<0.001$ ) in univariable analyses (Table 3). Statin use was not associated with RFS (HR: 1.75, 95\%-CI: 0.91-3.37, $\mathrm{p}=0.09)$.

Regression parameters from multivariable models evaluating the independent prognostic utility of LDL cholesterol (continuous and $3 \mathrm{mM}$ cut-point) are presented in Table 4. LDL cholesterol remained an independent prognostic factor in a multivariable models adjusting for age, PSA, extraprostatic disease, high-risk disease, positive nodal status, PSM and statin use. Table S2 shows cut-point testing for LDL cholesterol (1.5 to $5 \mathrm{mM})$. After correction for multiple comparisons, $3.5 \mathrm{mM}$ was another cut-point (besides $3.0 \mathrm{mM}$ ) that could predict time to BCR (HR: 0.38; 95\%-CI: 0.19-0.77; p=0.01; FDR-adjusted p-value: 0.03). Finally, KaplanMeier analysis in regard to BCR for the two LDL cholesterol cut-points (Figure 1: $3.0 \mathrm{mM}$, Figure 2: $3.5 \mathrm{mM}$ ) confirmed that the low LDL cholesterol subgroups were significantly associated with shorter time to BCR ( $\mathrm{p}=0.005$ for both cut-points). 


\section{Discussion}

Altered lipid metabolism has been acknowledged as a hallmark of many cancers including PCa $[15,16]$. Cancer cells exhibit increased lipid metabolism for energy generation and intracellular signaling, both of which are important for maintaining tumor cell survival [17]. Thus, there is a biological rationale for the investigation of dyslipidemia and lipid levels in cancer patients. Furthermore, epidemiologic studies have suggested that systemic metabolic disorders might be involved in PCa development and progression [18]. In this study we evaluated the prognostic role of preoperative serum lipid levels after a median follow-up of 28 months in 371 patients. Low LDL cholesterol remained an independent predictor for shorter RFS after RP for localized PCa. Other preoperative serum lipid serum levels (total cholesterol, HDL cholesterol and triglycerides) and statin use showed no significant association with the clinical course after RP in this cohort.

The main strength of this work is the prospective comprehensive evaluation of the complete preoperative lipid status consisting of the main serum lipid parameters and statin intake before RP. Only few studies in the past have evaluated the association of the complete preoperative serum lipid status and RFS after curative treatment for localized PCa. Regarding the prognostic role of LDL cholesterol conflicting results have been reported [7-10].

Two studies did not find any association between preoperative LDL cholesterol and outcome after RP for localized PCa. However, only one study [8] is comparable to ours. The cohort of the second study by Allot et al. [7] is characterized by almost $40 \%$ Gleason 6 cancer patients (compared to $13 \%$ in our cohort). Moreover, no information about the time point of blood sampling before surgery is available for this study. Two further studies reported pretreatment high LDL cholesterol levels to be associated with PCa recurrence, which contradicts our results $[9,10]$. Most patients in these two studies were treated with percutaneous radiotherapy and are thus not directly comparable to our work. Additionally, both studies have again reported a much higher amount of low risk PCa patients (between $44 \%$ up to $60 \%$ Gleason 6 
cancer) compared to our study. Finally, only a small subset of the entire cohort could be investigated for the complete pretreatment lipid levels (including LDL cholesterol) in one of the two studies [9]. In conclusion, different results have been reported regarding LDL cholesterol and its association with outcome after treatment of PCa. Heterogeneous cohorts with different populations and different therapies limit the comparison between the mentioned studies. To the best of our knowledge, our study is the first reporting preoperative low LDL cholesterol levels to be associated with PCa recurrence in men with localized PCa undergoing RP.

The role of low serum cholesterol on carcinogenesis is a hot topic in epidemiologic research, especially due to the high prevalence of cardiovascular morbidity and the resulting prescription of lipid-lowering agents such as statins [19-25].

One might explain the results of our investigation by the following hypothesis: Patients with preoperatively low LDL cholesterol levels may represent a group with higher and more aggressive tumor burden, which has the ability to alter the body's lipid metabolism leading to lower LDL cholesterol values and a higher risk for BCR. The essential question - as asked in a comprehensive Mendelian randomization study of Benn et al. [26] - is if the often independent association between low serum lipid levels and adverse oncologic outcomes is caused by a possible carcinogenic effect of low serum lipid levels or if we see a reverse causality, in which a preclinical malignancy alters the serum lipid metabolism. The study, which included over 10000 participants, concludes that low LDL cholesterol is probably secondary to preclinical cancer and that a causal relationship between serum lipid values and carcinogenesis is unlikely. Gilbert et al. demonstrated an inverse relationship between tumor mass of hematological cancers and serum cholesterol [27].Possible cell-biologic explanations for the reduction of LDL cholesterol in oncologic patients are the overutilization of serum lipids by cancer cells or the secretion of a humoral factor (by cancer cells) which stimulates normal cells to utilize LDL cholesterol [28-30]. A recent study has demonstrated that PCa 
cells require cholesterol for cell growth in vitro, thus it is reasonable to assume that faster growing tumors would consume more circulating LDL cholesterol [31]. In summary, the adverse role of low LDL cholesterol may be rather interpreted as a cause of cancer and may thus not represent a modifiable risk factor in preventing risk of $\mathrm{PCa}$ recurrence. As only preoperative serum lipid values were available for analysis, the dynamic changes of these parameters during the postoperative follow-up period, which might have yielded additional interesting results, were not included in this study, but should be included in future study protocols.

Studies from basic and translational research suggest a role for statins in PCa development or recurrence by several mechanisms [32]. In our study, statin use was not associated with PCa recurrence after RP. Conflicting results have been reported regarding statin use and PCa recurrence after curative treatment for localized disease. In line with our work, two relatively new meta-analyses that included patients undergoing RP or curative radiation therapy could not detect a significant association between statin use and PCa recurrence after RP $[33,34]$.

Our study has limitations: Firstly, the relatively short follow-up time, which led to a limited number of events. Secondly, only BCR and not PCa specific mortality was available as an oncological outcome parameter. BCR is a suboptimal oncological endpoint for PCa due to its rather limited ability to predict cancer-specific survival in PCa [35]. However, a shorter RFS is a surrogate marker for worse outcome in PCa after curative treatment [36,37]. Thirdly, other possible covariates/confounders of serum lipid status such as BMI or smoking status were not available for this study. Finally, the duration of preoperative statin intake was not controlled. However, the prediction of a shorter RFS by low LDL cholesterol was independent from statin intake in multivariable analyses. Our work provides a comprehensive blood analysis of all preoperative lipid serum levels in a consecutive series of PCa patients undergoing RP. 


\section{Conclusions}

This is the first study showing the potential prognostic role of low preoperative LDL cholesterol levels in a prospective cohort of patients with localized PCa undergoing RP. If long-term outcomes confirm our findings, preoperative low LDL cholesterol values might be used to categorize patients with localized PCa in a high-risk group for disease recurrence. 


\section{Acknowledgments}

The authors acknowledge Ms. Alexandra Veloudios for the excellent organization of the patient care during this study and Mr. Iakovos Amygdalos for English proofreading. This work was funded by a SystemsX.ch grant (Phosphonet-PPM) and a H2020 grant (PrECISE) to Peter Wild. 


\section{References}

[1] Wong MCS, Goggins WB, Wang HHX, Fung FDH, Leung C, Wong SYS, Ng CF, Sung JJY. Global Incidence and Mortality for Prostate Cancer: Analysis of Temporal Patterns and Trends in 36 Countries. Eur Urol 2016.

[2] Bill-Axelson A, Holmberg L, Garmo H, Rider JR, Taari K, Busch C, Nordling S, Häggman M, Andersson S-O, Spångberg A, Andrén O, Palmgren J, Steineck G, Adami H-O, Johansson J-E. Radical prostatectomy or watchful waiting in early prostate cancer. N Engl J Med 2014;370:932-42.

[3] Isbarn H, Wanner M, Salomon G, Steuber T, Schlomm T, Köllermann J, Sauter G, Haese A, Heinzer H, Huland H, Graefen M. Long-term data on the survival of patients with prostate cancer treated with radical prostatectomy in the prostate-specific antigen era. BJU Int 2010;106:37-43.

[4] Hackshaw-McGeagh LE, Perry RE, Leach VA, Qandil S, Jeffreys M, Martin RM, Lane JA. A systematic review of dietary, nutritional, and physical activity interventions for the prevention of prostate cancer progression and mortality. Cancer Causes Control CCC 2015;26:1521-50.

[5] Masko EM, Allott EH, Freedland SJ. The relationship between nutrition and prostate cancer: is more always better? Eur Urol 2013;63:810-20.

[6] YuPeng L, YuXue Z, PengFei L, Cheng C, YaShuang Z, DaPeng L, Chen D. Cholesterol Levels in Blood and the Risk of Prostate Cancer: A Meta-analysis of 14 Prospective Studies. Cancer Epidemiol Biomark Prev Publ Am Assoc Cancer Res Cosponsored Am Soc Prev Oncol 2015;24:1086-93.

[7] Allott EH, Howard LE, Cooperberg MR, Kane CJ, Aronson WJ, Terris MK, Amling CL, Freedland SJ. Serum lipid profile and risk of prostate cancer recurrence: Results from the SEARCH database. Cancer Epidemiol Biomark Prev Publ Am Assoc Cancer Res Cosponsored Am Soc Prev Oncol 2014;23:2349-56. 
[8] Kang M, Jeong CW, Ku JH, Kwak C, Kim HH. Hypertriglyceridemia is a potential preoperative predictor for biochemical recurrence after radical prostatectomy. PloS One 2015;10:e0122438.

[9] Gutt R, Tonlaar N, Kunnavakkam R, Karrison T, Weichselbaum RR, Liauw SL. Statin use and risk of prostate cancer recurrence in men treated with radiation therapy. J Clin Oncol Off J Am Soc Clin Oncol 2010;28:2653-9.

[10] Macleod LC, Chery LJ, Hu EYC, Zeliadt SB, Holt SK, Lin DW, Porter MP, Gore JL, Wright JL. Metabolic syndrome, dyslipidemia and prostate cancer recurrence after primary surgery or radiation in a veterans cohort. Prostate Cancer Prostatic Dis $2015 ; 18: 190-5$.

[11] Umbehr M, Kessler TM, Sulser T, Kristiansen G, Probst N, Steurer J, Bachmann LM. ProCOC: the prostate cancer outcomes cohort study. BMC Urol 2008;8:9.

[12] Friedewald WT, Levy RI, Fredrickson DS. Estimation of the concentration of lowdensity lipoprotein cholesterol in plasma, without use of the preparative ultracentrifuge. Clin Chem 1972;18:499-502.

[13] Executive Summary of The Third Report of The National Cholesterol Education Program (NCEP) Expert Panel on Detection, Evaluation, And Treatment of High Blood Cholesterol In Adults (Adult Treatment Panel III). JAMA 2001;285:2486-97.

[14] Benjamini Y, Hochberg Y. Controlling the false discovery rate: A practical and powerful approach to multiple testing. J R Stat Soc 1995;B:290-300.

[15] Butler LM, Centenera MM, Swinnen JV. Androgen control of lipid metabolism in prostate cancer: novel insights and future applications. Endocr Relat Cancer 2016.

[16] Zadra G, Photopoulos C, Loda M. The fat side of prostate cancer. Biochim Biophys Acta 2013;1831:1518-32.

[17] Ackerman D, Simon MC. Hypoxia, lipids, and cancer: surviving the harsh tumor microenvironment. Trends Cell Biol 2014;24:472-8. 
[18] Suburu J, Chen YQ. Lipids and prostate cancer. Prostaglandins Other Lipid Mediat 2012;98:1-10.

[19] Rose G, Blackburn H, Keys A, Taylor HL, Kannel WB, Paul O, Reid DD, Stamler J. Colon cancer and blood-cholesterol. Lancet Lond Engl 1974;1:181-3.

[20] Strasak AM, Pfeiffer RM, Brant LJ, Rapp K, Hilbe W, Oberaigner W, Lang S, Borena W, Concin H, Diem G, Ruttmann E, Glodny B, Pfeiffer KP, Ulmer H. Time-dependent association of total serum cholesterol and cancer incidence in a cohort of 172,210 men and women: a prospective 19-year follow-up study. Ann Oncol Off J Eur Soc Med Oncol ESMO 2009;20:1113-20.

[21] Law MR, Thompson SG. Low serum cholesterol and the risk of cancer: an analysis of the published prospective studies. Cancer Causes Control CCC 1991;2:253-61.

[22] Sherwin RW, Wentworth DN, Cutler JA, Hulley SB, Kuller LH, Stamler J. Serum cholesterol levels and cancer mortality in 361,662 men screened for the Multiple Risk Factor Intervention Trial. JAMA 1987;257:943-8.

[23] Neaton JD, Blackburn H, Jacobs D, Kuller L, Lee DJ, Sherwin R, Shih J, Stamler J, Wentworth D. Serum cholesterol level and mortality findings for men screened in the Multiple Risk Factor Intervention Trial. Multiple Risk Factor Intervention Trial Research Group. Arch Intern Med 1992;152:1490-500.

[24] Kritchevsky SB, Wilcosky TC, Morris DL, Truong KN, Tyroler HA. Changes in plasma lipid and lipoprotein cholesterol and weight prior to the diagnosis of cancer. Cancer Res $1991 ; 51: 3198-203$.

[25] Jacobs D, Blackburn H, Higgins M, Reed D, Iso H, McMillan G, Neaton J, Nelson J, Potter J, Rifkind B. Report of the Conference on Low Blood Cholesterol: Mortality Associations. Circulation 1992;86:1046-60.

[26] Benn M, Tybjla erg-Hansen A, Stender S, Frikke-Schmidt R, Nordestgaard BG. Lowdensity lipoprotein cholesterol and the risk of cancer: a mendelian randomization study. 
J Natl Cancer Inst 2011;103:508-19.

[27] Gilbert HS, Ginsberg H, Fagerstrom R, Brown WV. Characterization of hypocholesterolemia in myeloproliferative disease. Relation to disease manifestations and activity. Am J Med 1981;71:595-602.

[28] Kritz H, Zielinski C, Sinzinger H. Low cholesterol and cancer. J Clin Oncol Off J Am Soc Clin Oncol 1996;14:3043-8.

[29] Ho YK, Smith RG, Brown MS, Goldstein JL. Low-density lipoprotein (LDL) receptor activity in human acute myelogenous leukemia cells. Blood 1978;52:1099-114.

[30] Ueyama Y, Matsuzawa Y, Yamashita S, Funahashi T, Sakai N, Nakamura T, Kubo M, Tarui S. Hypocholesterolaemic factor from gallbladder cancer cells. Lancet Lond Engl 1990;336:707-9.

[31] Murtola TJ, Syvälä H, Pennanen P, Bläuer M, Solakivi T, Ylikomi T, Tammela TLJ. The importance of LDL and cholesterol metabolism for prostate epithelial cell growth. PloS One 2012;7:e39445.

[32] Papadopoulos G, Delakas D, Nakopoulou L, Kassimatis T. Statins and prostate cancer: molecular and clinical aspects. Eur J Cancer Oxf Engl 1990 2011;47:819-30.

[33] Scosyrev E, Tobis S, Donsky H, Wu G, Joseph J, Rashid H, Messing E. Statin use and the risk of biochemical recurrence of prostate cancer after definitive local therapy: a meta-analysis of eight cohort studies. BJU Int 2013;111:E71-7.

[34] Raval AD, Thakker D, Negi H, Vyas A, Salkini MW. Association between statins and clinical outcomes among men with prostate cancer: a systematic review and metaanalysis. Prostate Cancer Prostatic Dis 2016;19:151-62.

[35] Jhaveri FM, Zippe CD, Klein EA, Kupelian PA. Biochemical failure does not predict overall survival after radical prostatectomy for localized prostate cancer: 10-year results. Urology 1999;54:884-90.

[36] Freedland SJ. Screening, risk assessment, and the approach to therapy in patients with 
prostate cancer. Cancer 2011;117:1123-35.

[37] Sweeney C, Xie W, Williams SG. Disease-free survival (DFS) as a surrogate for overall survival (OS) in localized prostate cancer (CaP). J Clin Oncol 2016;34:(suppl; abstr 5023). 


\section{Figure legends}

Figure 1: Kaplan-Meier analysis and the corresponding log-rank test comparing time to biochemical recurrence between patients with low and high LDL cholesterol levels $(3.0 \mathrm{mM}$ cut-point).

Figure 2: Kaplan-Meier analysis and the corresponding log-rank test comparing time to biochemical recurrence between patients with low and high LDL cholesterol levels $(3.5 \mathrm{mM}$ cut-point). 